\title{
IL-17 promotes proliferation, inflammation and inhibits apoptosis of HaCaT cells via interacting with the TRAF3 interacting protein 2
}

\author{
JIE ZHANG ${ }^{1}$, JUNBIN ZHANG ${ }^{2}$, QINGXIA LIN $^{3}$, LAMEI CHEN $^{1}$ and YALI SONG ${ }^{1}$ \\ ${ }^{1}$ Department of Dermatology, Shandong Provincial Hospital Affiliated to Shandong University, \\ Jinan, Shandong 250021; ${ }^{2}$ Department of Dermatology, Affiliated Hospital of Shandong University of \\ Traditional Chinese Medicine, Jinan, Shandong 250014; ${ }^{3}$ Department of Dermatology, Affiliated Hospital of \\ Weifang Medical University, Weifang, Shandong 261000, P.R. China
}

Received February 23, 2020; Accepted September 18, 2020

DOI: $10.3892 / \mathrm{etm} .2020 .9480$

\begin{abstract}
The present study aimed to investigate the effects of interleukin-17 (IL-17) on the function of keratinocytes and to further investigate its associated mechanism. Human immortalized epidermal cells ( $\mathrm{HaCaT})$ were divided into sham control group (Sham), TRAF3 interacting protein 2 (TRAF3IP2)-knockdown with lentivirus group (si-TRAF3IP2), sham control+IL-17 group (Sham+IL-17) and TRAF3IP2-knockdown with lentivirus+IL-17 group (si-TRAF3IP2+IL-17). MTT and flow cytometry assays demonstrated that IL-17 promoted proliferation and inhibited apoptosis of $\mathrm{HaCaT}$ cells, while this effect was reversed following knockdown of TRAF3IP2 with lentiviral vectors. In addition, a marked increase in the levels of IL-6, IL-8, IL-23, TNF- $\alpha$ and VEGF was observed in the Sham+IL-17 group compared with that noted in the Sham group $(\mathrm{P}<0.05)$. Furthermore, reverse transcription-quantitative polymerase chain reaction and western blotting indicated that the mRNA and protein expression levels of caspase- 3 in the si-TRAF3IP2+IL-17 group were significantly increased compared with those of the Sham+IL-17 group $(\mathrm{P}<0.05)$. Taken together, the results indicated that IL-17 promoted proliferation and inflammation and inhibited apoptosis of $\mathrm{HaCaT}$ cells by interacting with the TRAF3IP2 adaptor protein, while knockdown of the expression of TRAF3IP2 reduced the effects of IL-17 in HaCaT cells.
\end{abstract}

Correspondence to: Dr Yali Song, Department of Dermatology, Shandong Provincial Hospital Affiliated to Shandong University, 324 Jingwu Road, Jinan, Shandong 250021, P.R. China

E-mail: songyali9@163.com

Key words: psoriasis, interleukin-17, proliferation, apoptosis, inflammation, TRAF3 interacting protein 2

\section{Introduction}

Psoriasis is a chronic inflammatory skin disease with a long course and a tendency to relapse. Various factors, including bacterial infection, and immune-mediated, genetic and environmental factors serve an important role in the development of psoriasis (1). In the general population, the incidence of psoriasis ranges between 0.6 and $4.8 \%$ (2). The clinical manifestations of psoriasis are red scaly plaques that can occur on any part of the body (3). Disfiguration, disability and associated comorbidity affect the physical health and mental status of the patients considerably (4). Therefore, the development of novel therapeutic strategies is imperative for the successful treatment of psoriasis.

Interleukin-17 (IL-17) is an inflammatory cytokine with diverse roles in immune protection and immunopathology $(5,6)$. IL-17 promotes the activation of T cells and stimulates epithelial cells, endothelial cells and fibroblasts to produce a variety of cytokines, which leads to inflammation (7). However, during abnormal expression or overexpression of IL-17, chronic inflammation is caused that is associated with several inflammatory disorders and autoimmune diseases (8). IL-17 participates in the occurrence and development of various immune diseases and inflammatory conditions by binding to the IL-17 receptor. This causes signal transduction and the induction of pro-inflammatory factors in order to mediate defense responses (9). Therefore, IL-17 is considered an attractive therapeutic target.

Tumor necrosis factor (TNF) receptor-associated factors (TRAFs) are key regulatory proteins in the TNF receptor-associated signaling pathway. They are characterized by a helical coiled structure and a conserved TRAF domain at the C-terminus (10). TRAF3 is one of the most versatile members of the TRAF family. It is an intracellular protein that primarily acts as an adaptor protein with a large variety of binding partners (11). TRAF3 interacting protein 2 (TRAF3IP2), also called CIKS or Act1, is a key intermediate in the normal inflammatory response and is involved in the pathogenesis of various autoimmune and inflammatory diseases (12). Furthermore, TRAF3IP2 is a protein acting downstream of the IL-17 receptor that binds to and stabilizes mRNAs encoding key inflammatory proteins (9). 
Human keratinocytes account for $>95 \%$ of human epidermal cells. Human immortalized epidermal cells (HaCaT) cells are immortalized cells with human keratinocyte differentiation and proliferation characteristics that have been widely used for studies involving skin biology and differentiation (13). Therefore, the present study aimed to investigate the effects of IL-17 on the proliferation, apoptosis and inflammation of $\mathrm{HaCaT}$ cells and to further investigate its mechanism of action. The experiments aimed to identify novel molecular targeted drugs or therapeutic targets that may effectively treat psoriasis.

\section{Materials and methods}

Cell culture. HaCaT cells were purchased from the Shanghai Institute of Cell Biology, Chinese Academy of Sciences. The cells were cultured in Dulbecco's modified Eagle's medium (Gibco; Thermo Fisher Scientific, Inc.) containing 10\% fetal bovine serum (Beijing Solarbio Science \& Technology Co., Ltd.) and $1 \%$ penicillin-streptomycin (Beijing Solarbio Science \& Technology Co., Ltd.) at $37^{\circ} \mathrm{C}$, in the presence of $5 \% \mathrm{CO}_{2}$. Growth phase cells were used for subsequent experiments.

Cell transfection and grouping. HaCaT cells in the logarithmic growth phase were adjusted to a density of $5 \times 10^{5}$ cells $/ \mathrm{ml}$ with trypsin and incubated in a 6 -well plate. When the cells reached $80 \%$ confluence, transfection was performed according to manufacturer's protocols of the lip2000 lentiviral transfection kit (Shanghai Jikai Biotechnology Co., Ltd.). The cells were divided into the 5 following groups: i) sham control group (Sham), which included cells transfected with an empty vector (Shanghai Jikai Biotechnology Co., Ltd.); ii) lentiviral-mediated TRAF3IP2-knockdown group (si-TRAF3IP2; 10/250 $\mu \mathrm{l}$ ), which included cells transfected with si-TRAF3IP2 (Shanghai Jikai Biotechnology Co., Ltd.); iii) sham control + IL-17 group (Sham + IL-17), which included cells transfected with empty carrier and cultured with $10 \mathrm{ng} / \mathrm{ml}$ IL-17A; iv) lentiviral-mediated TRAF3IP2-knockdown+IL-17 group (si-TRAF3IP2+IL-17), which included cells transfected with si-TRAF3IP2 and cultured with $10 \mathrm{ng} / \mathrm{ml} \mathrm{IL-17A}$; and v) negative control siRNA group (si-NC), which included cells transfected with scrambled siRNA (Shanghai Jikai Biotechnology Co., Ltd). All cell groups were cultured for $48 \mathrm{~h}$. The si-NC group was used as the transfection control. The target sequence of si-TRAF3IP2 was 5'-CCCCAAATACAAACAGGACGT-3'. The carrier frame structure of si-TRAF3IP2 was GV248 hU6-MCS-Ubiquitin-EGFP-IRES-puromycin. The si-NC sense strand was 5'-UUCUCCGAACGUGUCACGUTT-3' and antisense strand was 5'-ACGUGACACGUUCGGAGA ATT-3'.

MTT assay. A total of $100 \mu 1$ of cells were incubated in 96-well plates. The cells were incubated for 24,36, 48 and $72 \mathrm{~h}$ following transfection and IL-17 administration, and $100 \mu \mathrm{g}$ MTT was added. The cells were incubated at $37^{\circ} \mathrm{C}$ for $4 \mathrm{~h}$ and the supernatant was dissolved with $200 \mu$ l dimethyl sulfoxide (Sigma-Aldrich; Merck KGaA). A plate reader (Bio-Rad Laboratories, Inc.) was used to assess the optical density of each well at $495 \mathrm{~nm}$. The relative absorbance of each group was calculated by the following equation:

Relative absorbance $=\left(\mathrm{OD}_{\text {Experiment }}-\mathrm{OD}_{\text {Blank }}\right) /\left(\mathrm{OD}_{\text {Control }}-\mathrm{OD}_{\text {Blank }}\right)$

Flow cytometry. The cells in the different groups were collected and resuspended with pre-chilled PBS (1X) and centrifuged at $11,180 \mathrm{x} \mathrm{g}$ for $5 \mathrm{~min}$ at $4^{\circ} \mathrm{C}$. Following washing twice with precooled $\left(4^{\circ} \mathrm{C}\right)$ sterile PBS solution, the cells were fixed overnight with $70 \%$ ethanol and incubated at $4^{\circ} \mathrm{C}$. Following another wash with PBS, $100 \mu 1$ Rnase A was added to resuspend the cells. The cells were heated in a waterbath at $37^{\circ} \mathrm{C}$ for $30 \mathrm{~min}$. Subsequently, $400 \mu \mathrm{l}$ propidium iodide (PI) was added and the samples were incubated in the dark for $30 \mathrm{~min}$. The cell cycle was detected using a flow cytometer (Gallios; Beckman Coulter, Inc.). The AnnexinV-FITC apoptosis assay kit (BD Biosciences) was used for apoptosis detection. Following washing, $100 \mu \mathrm{l}$ binding buffer (1X) was added to the cells, and the resulting mixture was incubated with $5 \mu \mathrm{l}$ Annexin V-FITC in the dark for $15 \mathrm{~min}$. Subsequently, $10 \mu \mathrm{l}$ PI was added and mixed with the samples. The CellQuest 5.1 software (BD Biosciences) was used to analyze the data.

ELISA. The cell supernatants were collected and the levels of IL-6 (cat. no. HEA079Hu03; v.), IL-8 (cat. no. SEA080Si03; Saihongrui Technology Co., Ltd.), IL-23 (cat. no. SEA384Mu02; Saihongrui Technology Co.,Ltd.), TNF- $\alpha$ (cat. no.E-EL-M0049c; Elabscience Biotechnology Inc.) and VEGF (cat. no. GRJ13-009; Guangrui Biotechnology Co., Ltd.) in the cells were detected in strict accordance with the manufacturer's protocols of the ELISA kit. The optical densities at $450 \mathrm{~nm}$ were measured using a microplate reader (RT-6100; Lei Du).

Reverse transcription-quantitate polymerase chain reaction $(R T-q P C R)$. The cells in the different groups were collected and centrifuged at $4^{\circ} \mathrm{C}$, at $12,000 \mathrm{rpm}$ for $15 \mathrm{~min}$. Total RNA was extracted using the TRIzol reagent (Takara Bio, Inc.). The cDNA was synthesized using the reverse transcription kit (Applied Biosystems; Thermo Fisher Scientific, Inc.), according to the manufacturer's protocol. The SYBR-Green PCR kit (Qiagen, Inc.) was used as a fluorophore. The RT-qPCR assay was performed in a Mastercycler ${ }^{\circledR}$ nexus X2 (Eppendorf) using $2 \mu \mathrm{l}$ cDNA as a template under the following conditions: $95^{\circ} \mathrm{C}$ for $10 \mathrm{~min}, 95^{\circ} \mathrm{C}$ for $15 \mathrm{sec}$, and $60^{\circ} \mathrm{C}$ for $1 \mathrm{~min}$ for 40 cycles. The data were processed using the $2^{-\Delta \Delta \mathrm{Cq}}$ method (14) and the relative expression levels were calculated using GAPDH mRNA as an internal reference. The sequences of the primers (Shanghai Sheng gong Bioengineering Technology Service Co., Ltd.) used were as follows: TRAF3IP2 forward, 5'-CTGCGTCTG AGTCTGTGGTT-3' and reverse, 5'-TATCCCGTGTCTATG GTTGG-3'; caspase-3 forward, 5'-ATGGAGAACAATAAA ACCT-3' and reverse, 5'-CTAGTGATAAAAGTAGAGTTC-3'; GAPDH forward, 5'-TGACTTCAACAGCGACACCCA-3' and reverse, 5'-CACCCTGTTGCTGTAGCCAAA-3'.

Western blot analysis. The cells were lysed in RIPA buffer (Beyotime Institute of Biotechnology) and the protein concentration was measured using the bicinchoninic acid kit (Beijing Solarbio Science \& Technology Co., Ltd.). Each protein sample $(\sim 40 \mathrm{~g} /$ lane $)$ was separated on $10 \%$ sodium 


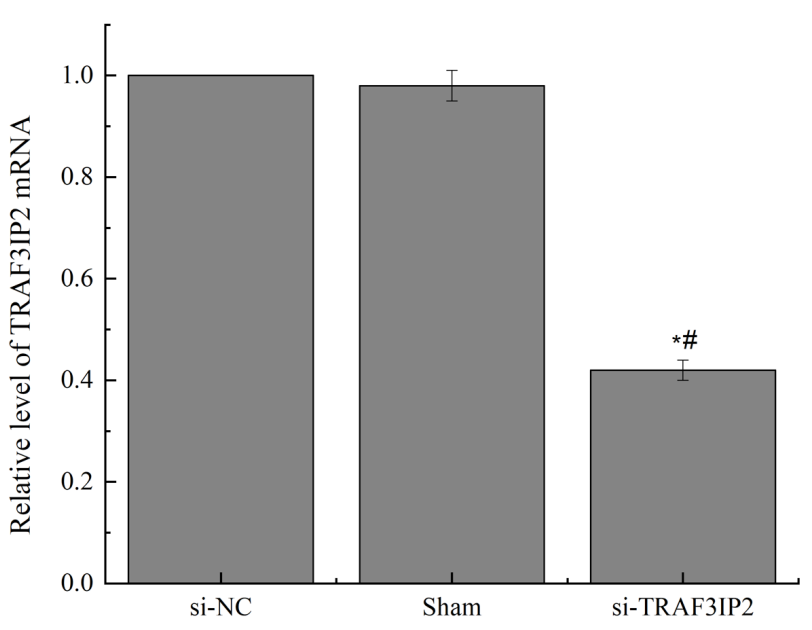

Figure 1. The mRNA expression of TRAF3IP2 in different groups. The values are indicative of the mean \pm standard deviation. ${ }^{*} \mathrm{P}<0.05$ compared with the si-NC group; ${ }^{\#} \mathrm{P}<0.05$ compared with the Sham group. si, small interfering RNA; NC, negative control; Sham, Sham control group; TRAF3IP2, TRAF3 interacting protein 2 .

dodecyl sulfate polyacrylamide gels by electrophoresis (Mini-protean-3; Bio-Rad Laboratories, Inc.) and transferred onto polyvinylidene difluoride membranes (Merck \& Co., Inc.). Subsequently, the membranes were blocked with 5\% skimmed milk and incubated overnight at $4^{\circ} \mathrm{C}$ with primary rabbit anti-human antibodies against caspase-3 (dilution, 1:1,000; cat. no. ab13847; Abcam) and GAPDH (dilution, 1:1,000; cat. no. ab70699; Abcam). The following day, horseradish peroxidase-conjugated goat anti-rabbit immunoglobulin $\mathrm{G}$ secondary antibody (dilution, 1:1,000; cat. no. ABIN101988; Antibodies Online) was used at room temperature for $1 \mathrm{~h}$. The membranes were observed and recorded using the enhanced chemiluminescence system (ImageQuant LAS 4000; General Electric Company) for 3-5 min. Protein expression levels were normalized to GAPDH, scanned and quantified using the ImageJ 1.46r software (National Institutes of Health).

Statistical analysis. All experiments were repeated at least three times. Statistical analysis was performed using SPSS 19.0 (IBM Corp.). The mean \pm standard deviation was used to measure the data. Significant differences between two groups were assessed using a Student's t-test. Pairwise comparisons were performed using the one-way analysis of variance test, followed by Tukey's post hoc test. $\mathrm{P}<0.05$ was considered to indicate a statistically significant difference.

\section{Results}

Expression of TRAF3IP2 $m R N A$ in different groups. The transfection efficiency was detected by RT-qPCR (Fig. 1). The TRAF3IP2 mRNA expression in the si-NC and Sham groups were not significantly different $(\mathrm{P}>0.05)$. By contrast, TRAF3IP2-knockdown using a lentivirus significantly decreased the expression of TRAF3IP 2 mRNA $(\mathrm{P}<0.05)$. This result indicated that the transfections were successful.

Effects of IL-17 on the proliferation of HaCaT cells. Cell proliferation ability was detected by MTT assays (Fig. 2). The

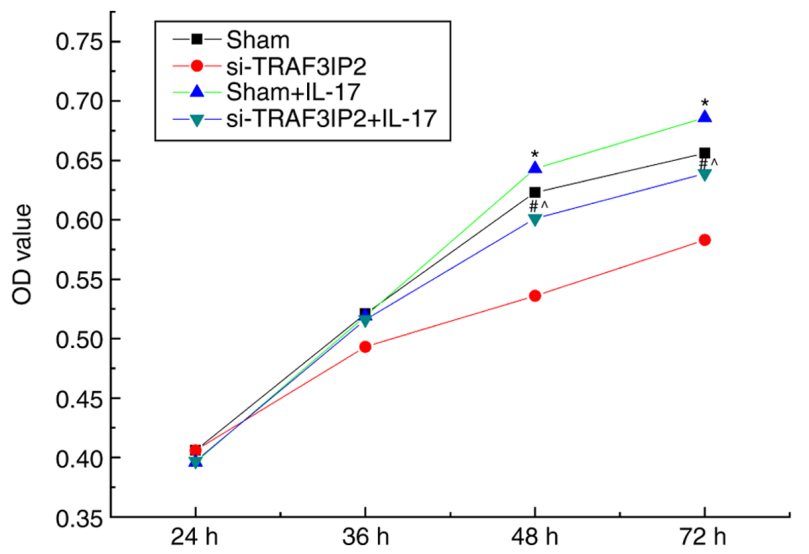

Figure 2. The OD values of different groups. The values are indicative of the mean \pm standard deviation. ${ }^{*} \mathrm{P}<0.05$ compared with the Sham group; ${ }^{\#} \mathrm{P}<0.05$ compared with the si-TRAF3IP 2 group; ${ }^{\wedge} \mathrm{P}<0.05$ compared with the Sham+IL-17 group. OD, optical density; Sham, Sham control group; si, small interfering RNA; TRAF3IP2, TRAF3 interacting protein 2; IL-17, interleukin-17.

si-TRAF3IP2 HaCaT cell group exhibited decreased proliferation compared with the Sham control cell group. On the other hand, in si-TRAF3IP2 HaCaT cells treated with IL-17 (si-TRAF3IP2 + IL-17), the cell proliferation was significantly decreased compared with the Sham control cell group $(\mathrm{P}<0.05)$. Furthermore, HaCaT cells treated with IL-17 exhibited increased proliferation compared with the Sham group and si-TRAF3IP2 + IL-17 group. In addition, compared with the Sham + IL-17 group, the proliferation ability was significantly decreased in the si-TRAF3IP2 + IL-17 group $(\mathrm{P}<0.05)$. These findings suggested that IL-17 promoted the proliferation of $\mathrm{HaCaT}$ cells by interacting with the TRAF3IP 2 adaptor protein.

Effects of IL-17 on the cell cycle of HaCaT cells. The percentage of cells in the G1 phase of the cell cycle in the Sham+IL-17 group $(58.32 \pm 3.21 \%)$ was significantly higher than that of the Sham group (Fig. 3; 54.18 $\pm 3.19 \%$; $\mathrm{P}<0.05$ ). However, the percentage of cells in the G1 phase of the si-TRAF3IP2+IL-17 group was decreased to $50.88 \pm 2.36 \%$, which was significantly lower than that in the Sham+IL-17 group $(\mathrm{P}<0.05)$. The results further indicated that IL-17 promoted the transition of the cell cycle from the $\mathrm{G} 1$ to the $\mathrm{S}$ phase and the proliferation of HaCaT cells via interacting with the TRAF3IP2 adaptor protein. Knockdown of TRAF3IP2 expression decreased the efficacy of IL-17 on the proliferation of HaCaT cells.

Effects of IL-17 on the induction of apoptosis of HaCaT cells. Flow cytometric analysis was used to detect the induction of cell apoptosis (Fig. 4). The number of apoptotic cells in the Sham+IL-17 group $(4.40 \pm 0.19 \%)$ was significantly lower than that in the Sham group $(5.02 \pm 0.23 \%$; $\mathrm{P}<0.05)$. However, the percentage of apoptotic cells in the si-TRAF3IP2+IL-17 group $(6.83 \pm 0.32 \%)$ was significantly higher than that in the Sham group $(\mathrm{P}<0.05)$, indicating that IL-17 inhibited the apoptosis of $\mathrm{HaCaT}$ cells by interacting with the TRAF3IP2 adaptor protein. Knockdown of the expression of TRAF3IP2 decreased the efficacy of IL-17 on the induction of apoptosis of HaCaT cells.

Effects of IL-17 on the levels of inflammatory factors in HaCaT cells. The cell supernatant cytokine levels were 

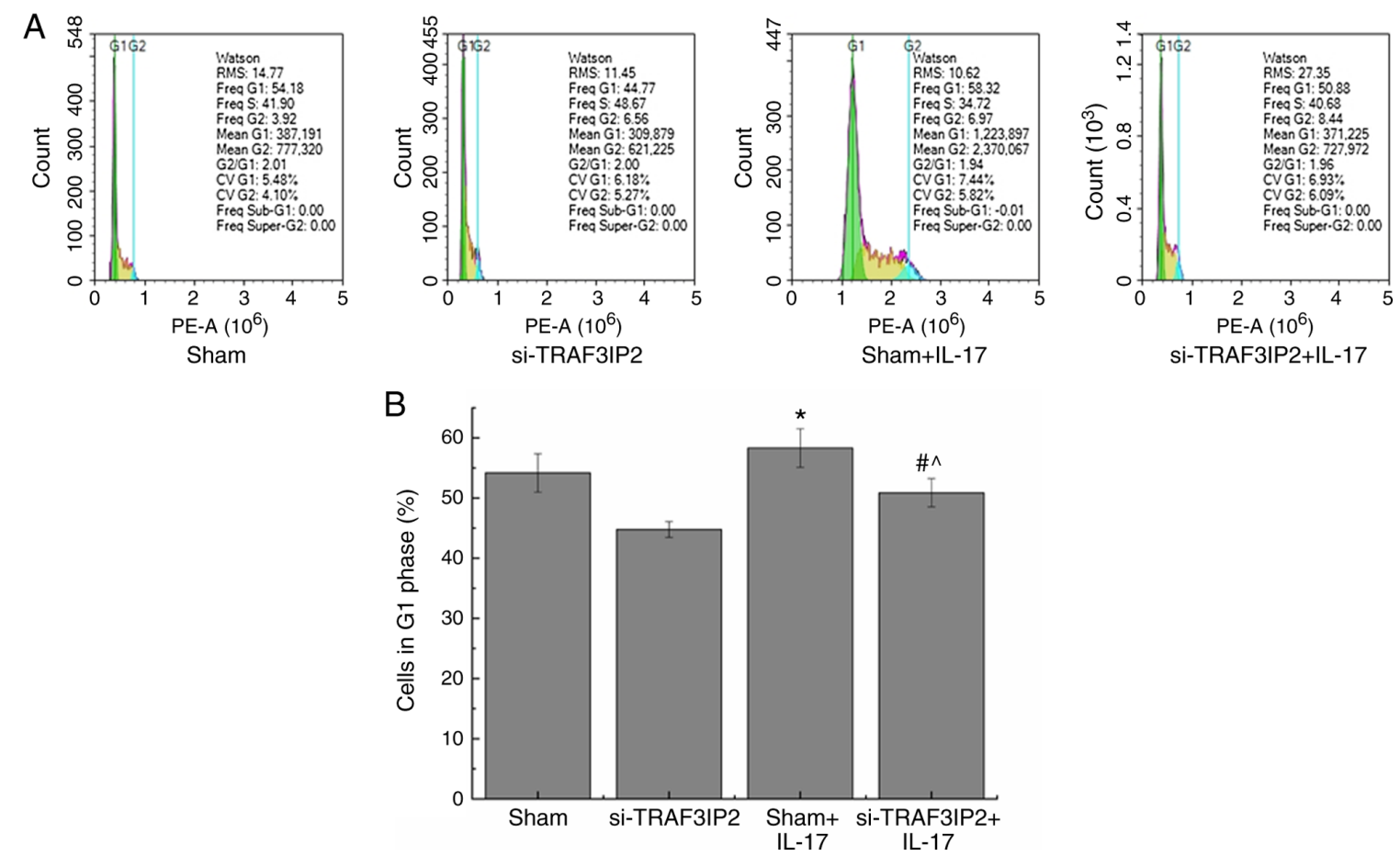

Figure 3. Cell cycle analysis of different cell groups. (A) Cell cycle, (B) Cells in G1 phase (\%). The values are indicative of the mean \pm standard deviation. "P $<0.05$ compared with the Sham group; ${ }^{~} \mathrm{P}<0.05$ compared with the si-TRAF3IP2 group; ${ }^{\wedge} \mathrm{P}<0.05$ compared with the Sham+IL-17 group. Sham, Sham control group; si, small interfering RNA; TRAF3IP2, TRAF3 interacting protein 2; IL-17, interleukin-17.
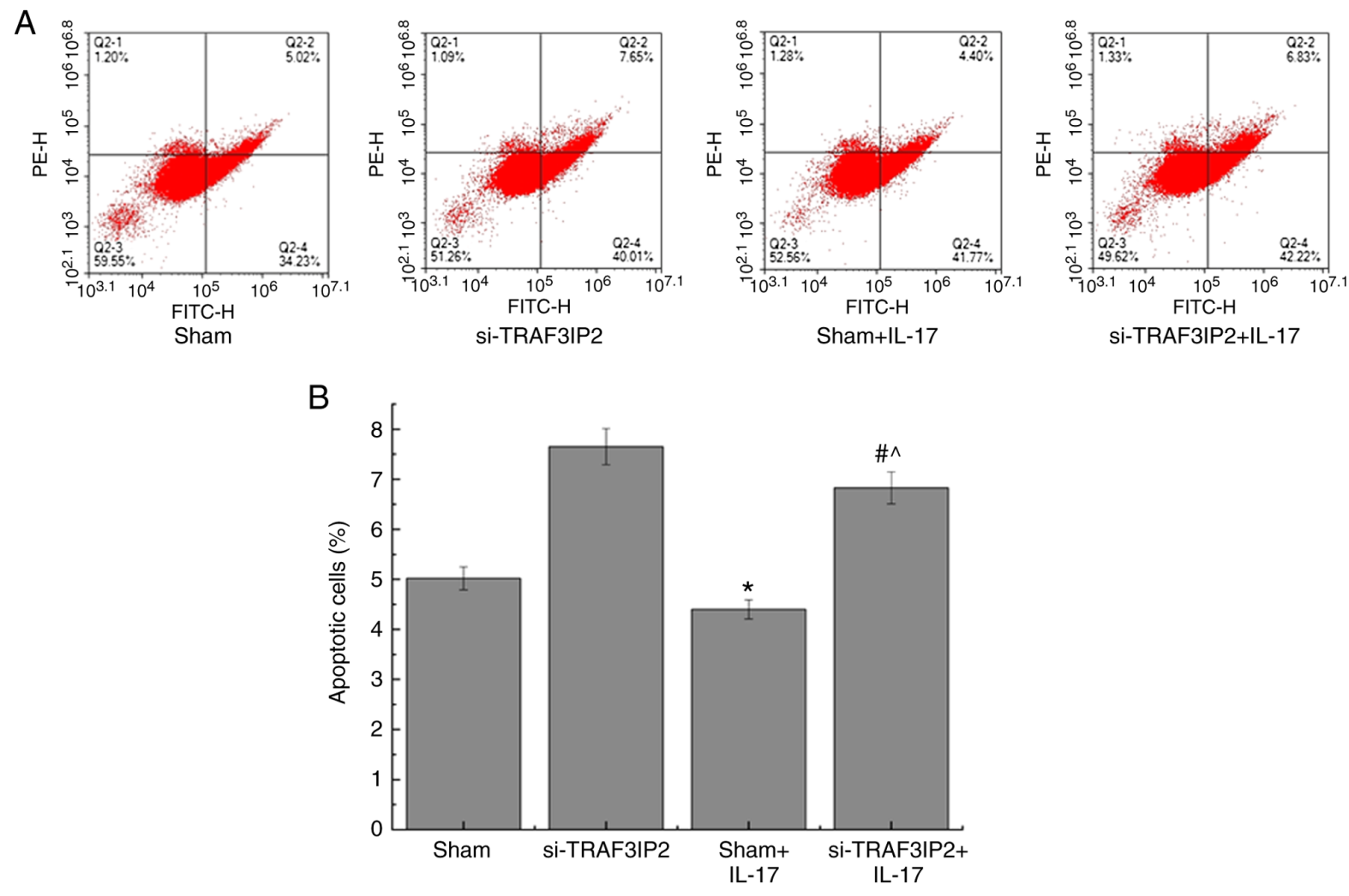

Figure 4. Changes in the percentage of apoptotic cells noted in the different groups. (A) Flow cytometry. (B) Apoptotic cells (\%). The values are mean \pm standard deviation. ${ }^{*} \mathrm{P}<0.05$ compared with Sham group; ${ }^{*} \mathrm{P}<0.05$ compared with si-TRAF3IP2 group; ${ }^{\wedge} \mathrm{P}<0.05$ compared with Sham+IL-17 group. Sham, Sham control group; si, small interfering RNA; TRAF3IP2, TRAF3 interacting protein 2; IL-17, interleukin-17.

analyzed in order to reflect the inflammatory response. The expression levels of IL-6, IL-8, IL-23, TNF- $\alpha$ and
VEGF were significantly increased in the Sham+IL-17 groups compared with those in the Sham group (Fig. 5; 

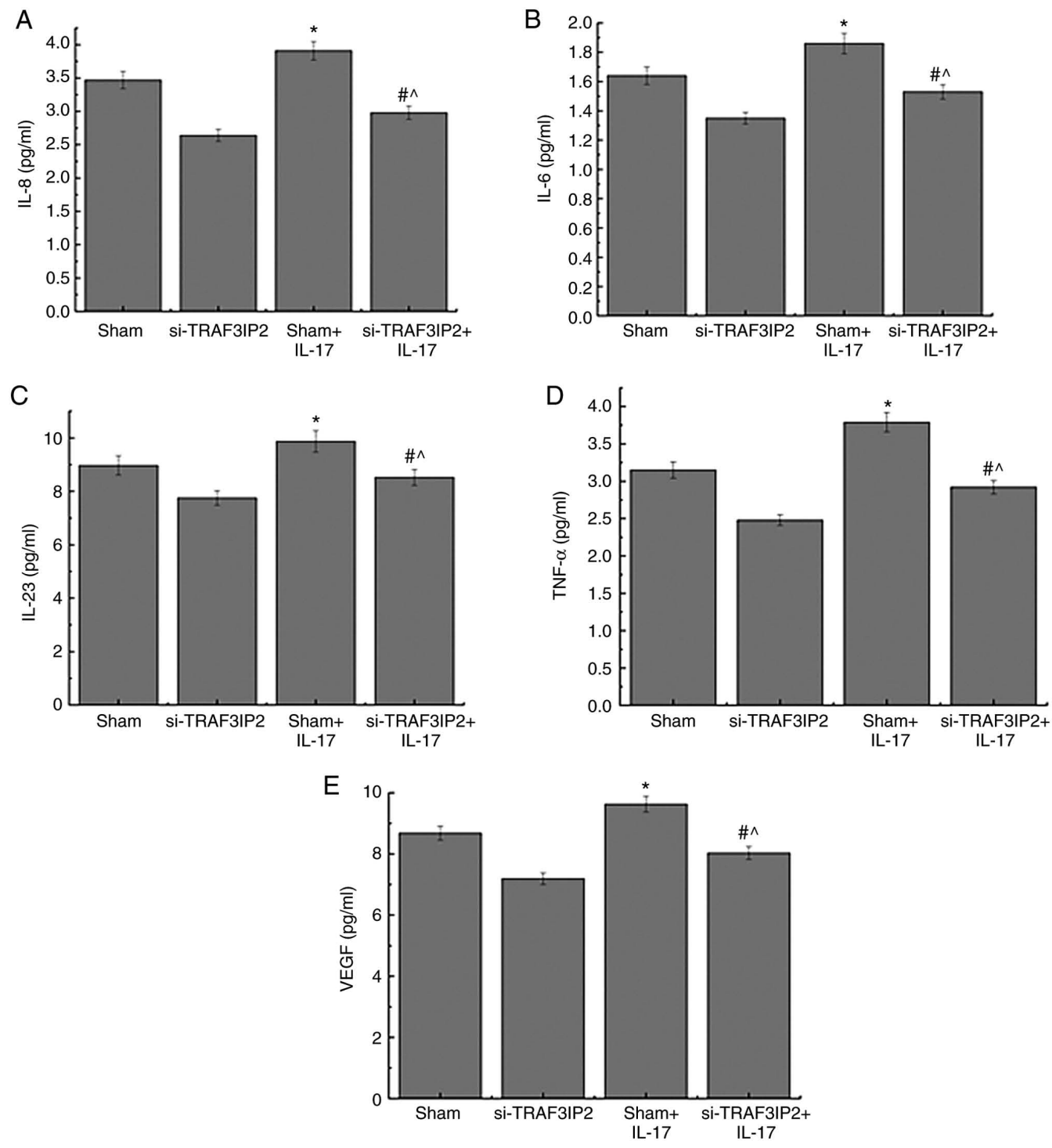

Figure 5. The levels of the inflammatory factors in the different groups. (A) IL-8. (B) IL-6. (C) IL-23. (D) TNF- $\alpha$. (E) VEGF. The values are indicative of the mean \pm standard deviation. ${ }^{*} \mathrm{P}<0.05$ compared with the Sham group; ${ }^{*} \mathrm{P}<0.05$ compared with the si-TRAF3IP2 group; ${ }^{\wedge} \mathrm{P}<0.05$ compared with the Sham+IL-17 group. IL, interleukin; TNF, tumor necrosis factor; VEGF, vascular endothelial growth factor; Sham, Sham control group; si, small interfering RNA; TRAF3IP2, TRAF3 interacting protein 2.

$\mathrm{P}<0.05)$. However, significant decreases in the levels of IL-6, IL-8, IL-23, TNF- $\alpha$ and VEGF were observed in the si-TRAF3IP2+IL-17 group compared with those in the Sham+IL-17 groups $(\mathrm{P}<0.05)$. These findings indicated that IL-17 promoted the inflammation of $\mathrm{HaCaT}$ cells by interacting with the TRAF3IP2 adaptor protein, while knockdown of TRAF3IP2 expression decreased the inflammatory efficacy of IL-17 on HaCaT cells.

Effects of IL-17 on the expression levels of caspase-3 in $\mathrm{HaCaT}$ cells. RT-qPCR and western blot analyses were used to investigate the mRNA and protein expression levels of caspase-3 in HaCaT cells, respectively (Fig. 6). The mRNA and protein expression levels of caspase-3 were decreased in the Sham+IL-17 group compared with those in the Sham group. Furthermore, a significant increase in the mRNA and protein expression levels of caspase- 3 was noted in the si-TRAF3IP2+IL-17 group compared with those of the Sham+IL-17 group. These results demonstrated that IL-17 decreased the expression levels of caspase- 3 by interacting with the TRAF3IP2 adaptor protein in HaCaT cells. These effects were reversed by lentiviral-mediated knockdown of TRAF3IP2.

\section{Discussion}

Psoriasis is a programmed pathological interaction among skin cells, and immune-mediated genetic and environmental factors (1). The hallmarks of psoriasis include deep-level epidermal modifications, including hyperproliferation and changes in keratinocyte differentiation, along with significant inflammatory cell infiltration and neovascularization (15). The concept of using anti-IL-17 as a potential treatment agent is not novel. The FDA approved secukinumab in 2015 and ixekizumab in 2016 to treat adults with moderate-to-severe plaque psoriasis (16). Furthermore, TRAF3IP2 is one of the genetic factors implicated in psoriasis that is involved in the IL23/IL-17 axis (17). Therefore, the present study investigated 


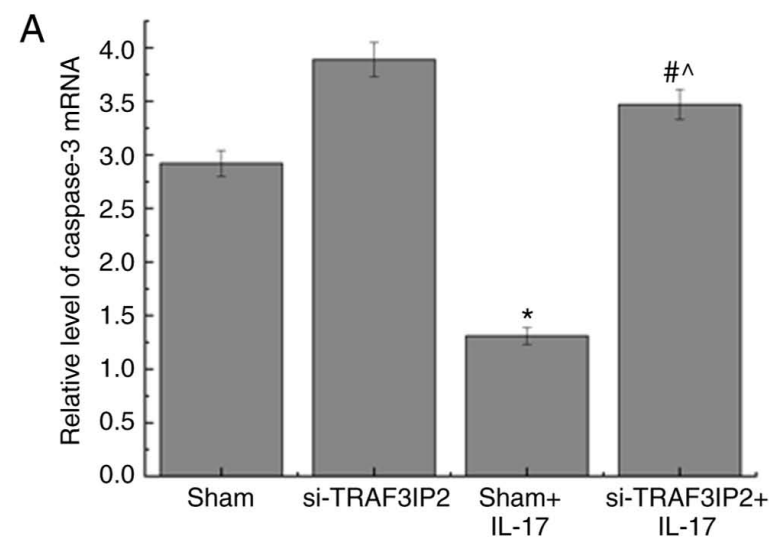

B
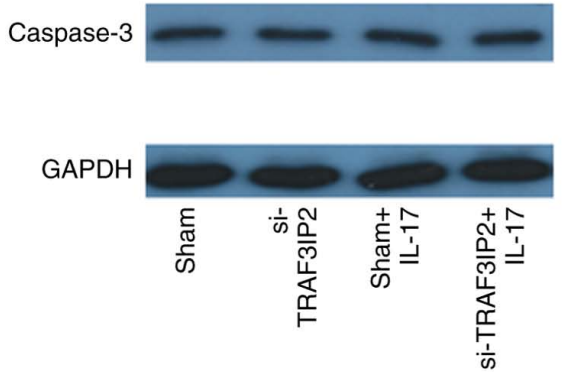

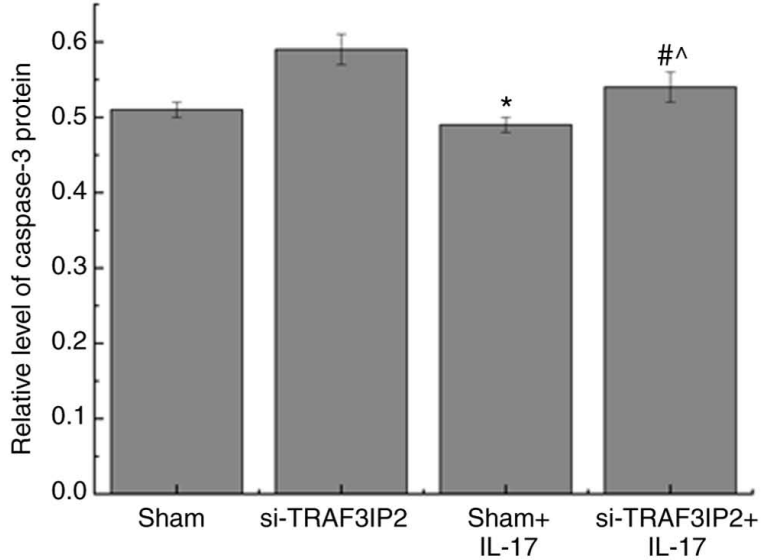

Figure 6. The mRNA and protein expression levels of caspase-3 in the different groups. (A) Reverse transcription-quantitative polymerase chain reaction. (B) Western blotting. The values are indicative of the mean \pm standard deviation. ${ }^{*} \mathrm{P}<0.05$ compared with the Sham group; ${ }^{\#} \mathrm{P}<0.05$ compared with the si-TRAF3IP2 group; ${ }^{\wedge} \mathrm{P}<0.05$ compared with the Sham+IL-17 group. Sham, Sham control group; TRAF3IP2, TRAF3 interacting protein 2; si, small interfering RNA; IL-17, interleukin-17.

the role of IL-17 as a key effector molecule on psoriasis and investigated its association with TRAF3IP2.

Cell proliferation is one of the important physiological functions of viable cells. However, several diseases, including psoriasis, are associated with abnormal cell proliferation, migration and invasion $(18,19)$. The results of the MTT assay indicated that IL-17 promoted the proliferation of $\mathrm{HaCaT}$ cells via interacting with the TRAF3IP2 adaptor protein. The complete cell cycle includes the G1, S and G2 phases and the mitotic phase (20).Lentiviral-mediated TRAF3IP2-knockdown can affect the cell cycle and cause abnormal arrest of the cells in the G1 phase, thereby reducing the number of cells entering the $S$ and $G 2$ phases and causing a reduction in the proliferation index. Apoptosis is a basic biological phenomenon of cells that is required to maintain the stability of the intracellular environment. The caspase family of enzymes is an important mediator of programmed cell death (apoptosis) and caspase- 3 is directly involved in the transduction of apoptotic signals $(21,22)$. In the present study, the apoptotic rate, and mRNA and protein expression levels of caspase- 3 were significantly increased in the IL-17+si-TRAF3IP2 group compared with those in the Sham+IL-17 group. These findings demonstrated that IL-17 promoted proliferation and inhibited apoptosis of HaCaT cells, while knockdown of TRAF3IP2 expression decreased the efficacy of IL-17.

Psoriasis is a chronic recurrent inflammatory skin disease that interacts with multiple factors, including immune-mediated genetic and environmental factors (1). The number of
Th17 cells is increased in the peripheral blood of patients with psoriatic disease and its effector IL-17 serves an important role in the severity of psoriasis (15). IL-17 stimulates the production of VEGF, IL-6, IL-8, granulocyte-macrophage colony stimulating factor, IL-23, TNF- $\alpha$ and other cytokines from macrophages $(23,24)$. VEGF is a highly specific vascular endothelial cell growth factor that promotes increased vascular permeability, extracellular matrix degeneration, and vascular endothelial cell migration, proliferation and angiogenesis (25). IL-6, IL-8, IL-23 and TNF- $\alpha$ are cytokines with multiple biological effects and are considered important inflammatory factors. Various cytokines can cause a chronic inflammatory state in skin cells and alter epidermal hyperproliferation, differentiation, apoptosis and neoangiogenesis that result in the production of skin diseases. In the present study, ELISA indicated that IL-17 promoted the inflammation of HaCaT cells by interacting with the TRAF3IP2 adaptor protein. Taken together, the results indicated that IL-17 served a crucial role in the pathogenesis and development of psoriasis and that knockdown of TRAF3IP2 may be considered a novel therapeutic target for psoriasis.

The present study demonstrated that IL-17 promoted proliferation and inflammation, while inhibiting the apoptosis of HaCaT cells. The possible mechanism of action may be via its interaction with the TRAF3IP2 adaptor protein. In addition, the data provided novel evidence on the role of TRAF3IP2 as a new therapeutic target for psoriasis. IL-17 may regulate proliferation, apoptosis and inflammation of human immortalized 
epidermal cells by interacting with the TRAF3IP2 adaptor protein. However, the lack of co-immunoprecipitation experiments to prove the hypothesis that IL-17 interacts with TRAF3IP2 is a limitation of the present study.

\section{Acknowledgements}

Not applicable.

\section{Funding}

The present study was supported by the National Natural Science Foundation of China Youth Fund (grant no. 81502729).

\section{Availability of data and materials}

The datasets used and/or analyzed during the current study are available from the corresponding author on reasonable request.

\section{Authors' contributions}

JiZ conducted the experiments, data collection and interpretation. YS participated in study design, coordination of the experiments and data collection. JuZ participated in the study design, data collection and data analysis and prepared the manuscript. QL and LC participated in study design, data analysis, data interpretation and wrote the manuscript. All authors read and approved the final version of the manuscript.

\section{Ethics approval and consent to participate}

The present study was approved by the Ethics Committee of Shandong Provincial Hospital Affiliated to Shandong University.

\section{Patient consent for publication}

Not applicable.

\section{Competing interests}

The authors declare that they have no competing interests.

\section{References}

1. Hugh JM and Weinberg JM: Update on the pathophysiology of psoriasis. Cutis 102: 6-12, 2018.

2. Naldi L: Epidemiology of psoriasis. Curr Drug Targets Inflamm Allergy 3: 121-128, 2004

3. Boehncke WH: Systemic inflammation and cardiovascular comorbidity in psoriasis patients: Causes and consequences. Front Immunol 9: 579, 2018.

4. Boehncke WH and Schön MP: Psoriasis. Lancet 386: 983-994, 2015.

5. Brembilla NC, Senra L and Boehncke WH: The IL-17 family of cytokines in psoriasis: IL-17A and beyond. Front Immunol 9 1682,2018
6. Amatya N, Garg AV and Gaffen SL: IL-17 signaling: The Yin and the Yang. Trends Immunol 38: 310-322, 2017.

7. Kuwabara T, Ishikawa F, Kondo $M$ and Kakiuchi T: The role of IL-17 and related cytokines in inflammatory autoimmune diseases. Mediators Inflamm 2017: 3908061, 2017.

8. Beringer A, Noack M and Miossec P: IL-17 in chronic inflammation: From discovery to targeting. Trends Mol Med 22: 230-241, 2016.

9. Herjan T, Hong L, Bubenik J, Bulek K, Qian W, Liu C, Li X, Chen X, Yang H, Ouyang S, et al: IL-17-receptor-associated adaptor Act1 directly stabilizes mRNAs to mediate IL-17 inflammatory signaling. Nat Immunol 19: 354-365, 2018.

10. Tang $X$, Zhang L and Wei W: Roles of TRAFs in NF- $\kappa B$ signaling pathways mediated by BAFF. Immunol Lett 196: 113-118, 2018.

11. Yi Z, Wallis AM and Bishop GA: Roles of TRAF3 in T cells: Many surprises. Cell Cycle 14: 1156-1163, 2015.

12. Erikson JM, Valente AJ, Mummidi S, Kandikattu HK, DeMarco VG, Bender SB, Fay WP, Siebenlist U and Chandrasekar B: Targeting TRAF3IP2 by genetic and interventional approaches inhibits ischemia/reperfusion-induced myocardial injury and adverse remodeling. J Biol Chem 292: 2345-2358, 2017

13. Deyrieux AF and Wilson VG: In vitro culture conditions to study keratinocyte differentiation using the HaCaT cell line. Cytotechnology 54: 77-83, 2007.

14. Livak KJ and Schmittgen TD: Analysis of relative gene expression data using real-time quantitative PCR and the 2(-Delta Delta C(T)) method. Methods 25: 402-408, 2001.

15. Sakkas LI and Bogdanos DP: Are psoriasis and psoriatic arthritis the same disease? The IL-23/IL-17 axis data. Autoimmun Rev 16: 10-15, 2017.

16. Campa M, Mansouri B, Warren R and Menter A: A review of biologic therapies targeting IL-23 and IL-17 for use in moderate-to-severe plaque psoriasis. Dermatol Ther (Heidelb) 6: 1-12, 2016.

17. Sukhov A, Adamopoulos IE and Maverakis E: Interactions of the immune system with skin and bone tissue in psoriatic arthritis: A comprehensive review. Clin Rev Allergy Immunol 51: 87-99, 2016.

18. Greb JE, Goldminz AM, Elder JT, Lebwohl MG, Gladman DD, Wu JJ, Mehta NN, Finlay AY and Gottlieb AB: Psoriasis. Nat Rev Dis Primers 2: 16082, 2016.

19. Iciek MB, Kowalczyk-Pachel D, Kwiecień I and Dudek MB: Effects of different garlic-derived allyl sulfides on peroxidative processes and anaerobic sulfur metabolism in mouse liver. Phytother Res 26: 425-431, 2012.

20. Wee P and Wang Z: Cell cycle synchronization of HeLa cells to assay EGFR pathway activation. Methods Mol Biol 1652: 167-181, 2017.

21. Fan TJ, Han LH, Cong RS and Liang J: Caspase family proteases and apoptosis. Acta Biochim Biophys Sin (Shanghai) 37: 719-727, 2005 .

22. Choudhary GS, Al-Harbi S and Almasan A: Caspase-3 activation is a critical determinant of genotoxic stress-induced apoptosis. Methods Mol Biol 1219: 1-9, 2015.

23. Coimbra S, Oliveira H, Reis F, Belo L, Rocha S, Quintanilha A, Figueiredo A, Teixeira F, Castro E, Rocha-Pereira $\mathrm{P}$ and Santos-Silva A: Interleukin (IL)-22, IL-17, IL-23, IL-8, vascular endothelial growth factor and tumour necrosis factor- $\alpha$ levels in patients with psoriasis before, during and after psoralen-ultraviolet A and narrowband ultraviolet B therapy. Br J Dermatol 163: 1282-1290, 2010

24. Furue K, Ito T and Furue M: Differential efficacy of biologic treatments targeting the TNF- $\alpha / \mathrm{IL}-23 / \mathrm{IL}-17$ axis in psoriasis and psoriatic arthritis. Cytokine 111: 182-188, 2018.

25. Melincovici CS, Boşca AB, Şuşman S, Mărginean M, Mihu C, Istrate M, Moldovan IM, Roman AL and Mihu CM: Vascular endothelial growth factor (VEGF)-key factor in normal and pathological angiogenesis. Rom J Morphol Embryol 59: 455-467, 2018.

This work is licensed under a Creative Commons Attribution-NonCommercial-NoDerivatives 4.0 International (CC BY-NC-ND 4.0) License. 